Revue internationale P.M.E.

Économie et gestion de la petite et moyenne entreprise

Revue

internationale

PME

\title{
Création d'entreprise et analyse du marché : une problématique à explorer
}

\section{Nathalie Schieb-Bienfait}

Volume 13, numéro 3-4, 2000

URI : https://id.erudit.org/iderudit/1008682ar

DOI : https://doi.org/10.7202/1008682ar

Aller au sommaire du numéro

Éditeur(s)

Presses de l’Université du Québec

ISSN

0776-5436 (imprimé)

1918-9699 (numérique)

Découvrir la revue

Citer cet article

Schieb-Bienfait, N. (2000). Création d'entreprise et analyse du marché : une problématique à explorer. Revue internationale P.M.E., 13(3-4), 89-117.

https://doi.org/10.7202/1008682ar
Résumé de l'article

Après avoir examiné comment la littérature de gestion aborde la question de l'analyse du marché dans le cadre de projets entrepreneuriaux, nous présentons deux projets de création d'entreprise qui ont fait l'objet d'un travail de recherche exploratoire où nous avons cherché à restituer de façon diachronique le processus d'approche et d'analyse du marché. L'étude des comportements et pratiques observés nous a permis d'inférer certaines remarques et de révéler quelques dissonances entre la variété et la diversité des problèmes et des situations rencontrés et le caractère réducteur des démarches dans lesquelles on veut contraindre les créateurs. Au regard de ces constats, nous avons cherché à formuler de nouvelles pistes de réflexion sur les spécificités et conditions d'efficacité des analyses du marché dans le cadre de la création d'entreprise. 


\title{
Création d'entreprise et analyse du marché: une problématique à explorer
}

Nathalie SCHIEB-BIENFAIT

IAE Université de Nantes

MOTS CLÉS

\section{Création d'entreprise - Marché - Analyse de marché \\ Créateur - Études de cas}

\begin{abstract}
RÉSUMÉ
Après avoir examiné comment la littérature de gestion aborde la question de l'analyse du marché dans le cadre de projets entrepreneuriaux, nous présentons deux projets de création d'entreprise qui ont fait l'objet d'un travail de recherche exploratoire où nous avons cherché à restituer de façon diachronique le processus d'approche et d'analyse du marché. L'étude des comportements et pratiques observés nous a permis d'inférer certaines remarques et de révéler quelques dissonances entre la variété et la diversité des problèmes et des situations rencontrés et le caractère réducteur des démarches dans lesquelles on veut contraindre les créateurs. Au regard de ces constats, nous avons cherché à formuler de nouvelles pistes de réflexion sur les spécificités et conditions d'efficacité des analyses du marché dans le cadre de la création d'entreprise.
\end{abstract}

\section{L'AUTEURE}

Maître de conférences en sciences de gestion à l'IAE (Institut d'administration des entreprises) de l'Université de Nantes, où elle est responsable du DESS Gestion et management des PME et chargée du développement de l'enseignement de l'entrepreneuriat. Docteure en sciences de gestion, diplômée de l'EDHEC et d'un DESS en système d'information (Université ParisDauphine), elle a travaillé plusieurs années dans le domaine du conseil en management. Elle est chercheure au sein du laboratoire Modélisaction, dirigé par le professeur $P$. Mevellec; ses travaux de recherche récents portent plus particulièrement sur les problématiques de création et développement d'activité, sur la stratégie des PME ainsi que sur l'enseignement de l'entrepreneuriat. Adresse: Rue de la Sensive du Tertre, B.P. 62232, 44322 Nantes, Cedex 3 , France, téléphone : 02401412 20, télécopieur: 0240746183 et courriel : <schieb-bienfait @iae.univ-nantes.fr> 


\begin{abstract}
After defining the meaning and scope of market analysis, we report and describe two case studies, which are the result of several months of clinical work on the business creation period. After studying how entrepreneurs really conduct their market analysis in the face of a set of new problems and constraints in different competitive environments, we discover that major models do not provide a comprehensive approach to the market analysis process observed. This research attempts to assess the possible contribution of more recent studies (particularly from a constructivist perspective). As a conclusion, we develop some new assumptions and discuss implications and directions for future research.
\end{abstract}

\title{
RESUMEN
}

Despues de haber examinado còmo la literatura de gestiòn aborda la cuestiòn del analisis dentro del plan de projectos empresariales. Nosotros presentamos dos projectos de creaciòn de empresas que lian sido objeto de un trabajo de analisis de exploraciòn donde liemos intentado restituir de ruanere progresiva el proceso de acercamiento anàlisis del mercado.

El estudio de comportamientos y pràcticas observados nos ha permitido alegar ciertas visiones sobre la forma con la cual los creadores de empresas construyen su visiòn y el conscimiento del mercado desde las primeras fases del elaboraciòn y puesta en servicio de su proyecto. Este trabajo revela las disonancias entre la variedad y diversidad de problemas y situaciones encontradas, el caracter normativo y lineal de gestiones habitualmente propuestas a los creadores.

Considerando estas observaciones nosotros proponemos un nuevo plan technico, paro la gestiòn de anàlisis de mercado por el creador. Dentro de este contexto formulamos varias proposiciones y pistas de reflexiòn sobre la especifidad $y$ condiciònes de eficacidad de los anàlisis de mercado dentro del plan de creaciòn de una empresa.

\section{ZUSAMMENFASSUNG}

Die Untersuchung der Managementliteratur über die Marktforschung bei der Gründung eines Unternehmens bildet die Ausgangslage, für die Präsentation von zwei Projekten der Unternehmensgründung.

Bei diesen zwei Forschungsprojekten haben wir versucht, die Art und Weise nachzuvollziehen, wie der Markt analysiert wird. Das Studium der diversen Verhaltensweisen und Praktiken hat aufgezeigt, dass keine Übereinstimmung zwischen der Theorie und der Vielfalt der angetroffenen Probleme besteht. Darauf aufbauend haben wir neue Ideen und Überlegungen formuliert, um die speziellen Bedingungen und die Effizienz von Marktuntersuchungen bei Unternehmensgründungen zu beschreiben. 


\section{Introduction}

Que l'on fasse référence à la connaissance du marchél par le créateur, aux perspectives d'évolution ou au positionnement du projet sur le marché, la problématique de l'analyse du marché est omniprésente dans le champ de la création d'entreprises (depuis l'émergence de l'idée jusqu'à la phase de démarrage et postconsolidation du projet), mais cette situation n'est pas exempte d'ambiguité.

L'expression "marché » pose d'emblée problème, car elle est souvent employée indifféremment pour parler de l'environnement global du projet, de l'environnement concurrentiel et du marché cible ${ }^{2}$. La notion d'analyse du marché peut donc recouvrir des réalités fort différentes, comme en témoignent les débats sur l'étude des projets entrepreneuriaux (MacMillan, 1985; Belley, Dussault et Lorrain, 1991 ; Copin et Marion, 1992). En analysant le discours et les pratiques sur l'analyse du marché dans ce contexte entrepreneurial, on découvre une problématique beaucoup plus complexe qu'il n'y paraît :

- d'une part, de nombreux articles et travaux témoignent de situations insatisfaisantes qui concernent aussi bien les défaillances des outils et méthodes préconisés que les pratiques engagées : en effet, les firmes nouvellement créées connaissent très mal leur marché (Marchesnay et Julien, 1988) et particulièrement lorsque le projet d'activité est novateur (Block et MacMillan, 1985); les créateurs entrepreneurs méconnaissent notamment la structure et les perspectives d'évolution de leur marché. Si quelques prospections sont généralement effectuées préalablement à la création, aucune étude approfondie n'est réalisée. Selon LaBarbera et Rosenberg (1989), l'évaluation des potentialités du marché est l'un des plus graves problèmes au moment du démarrage d'une entreprise. Ainsi, beaucoup d'entrepreneurs auraient tendance à surestimer la demande (Hills, 1985). D'autres auraient tendance à ignorer, voire à mettre de côté, l'information négative, contraire à leur projet, à privilégier leurs collectes d'information dans des secteurs familiers (Cooper, Folta et Woo, 1995) ou à procéder à certains types de recherches dans le seul but de satisfaire aux exigences de leur banquier. L'analyse du marché peut alors présenter les aspects d'une étude « alibi »;

1. Nous préférons employer le terme «marché » plutôt que celui d'environnement, car l'expression « marché » est plus couramment utilisée dans la littérature sur la création d'entreprise et dans les pratiques des créateurs.

2. En général, en stratégie, on préfère parler d'analyse de l'environnement pour renvoyer à l'ensemble des forces et dimensions dont le dirigeant doit tenir compte pour élaborer sa stratégie et réserver le terme d'analyse de marché à l'analyse de l'offre et de la demande dans une perspective plus marketing. 
- par ailleurs, les acteurs de l'univers de la création d'entreprise (boutiques de gestion, pépinières, société de capital risque, univers bancaire, etc.) éprouvent un certain embarras à nous dire explicitement ce qu'ils entendent par analyse du marché ; chacun considère l'analyse du marché comme primordiale, mais au regard de modalités et de critères qui lui sont propres.

Aujourd'hui, théoriciens et acteurs du milieu de l'entrepreneuriat s'accordent de plus en plus à reconnaître que l'analyse du marché ne renvoie pas à une connaissance objective, mais plus à un triptyque créateur-projet-approche du marché ; dès lors, ce n'est pas la description et la connaissance détaillée, documentée ou «livresque » du marché dont il est question, mais plutôt de la nature des relations et interactions entre ces trois composantes du triptyque.

C'est dans ce contexte que s'inscrit la présente recherche sur l'analyse du marché dans le cadre bien spécifique des projets entrepreneuriaux. À l'issue d'un travail exploratoire réalisé sur deux études de cas, nous nous interrogeons sur ce que doit être la démarche d'analyse de marché pour le créateur. À ce titre, nous formulons plusieurs questions de recherche, après avoir cherché à mieux comprendre les relations et interactions qui existent au sein du triptyque créateur-projet-marché.

Dans une première partie, nous précisons ce que l'on entend par marché et analyse du marché, dans le contexte précis d'une création d'entreprise. À cet effet, nous examinons comment ces questions sont traitées dans la littérature de gestion et quels types de problème elles soulèvent. Comment définir le marché de référence d'un projet et le périmètre d'analyse à retenir ? Quelles sont les dimensions d'analyse à prendre en considération ? Ce détour conceptuel nous permet d'introduire et de justifier le modèle ainsi que les dimensions d'analyse retenus pour étudier les deux cas.

Puis, dans une deuxième partie, sont présentés deux projets de création, à propos desquels nous avons cherché à observer et à comprendre les processus d'élaboration d'une vision et d'une connaissance du marché. Comment les créateurs ont-ils mené les démarches d'analyse du marché ? Quels sont les comportements et pratiques observés? L'interprétation des résultats poursuit un double objectif : restituer de façon diachronique le processus d'approche du marché et comprendre la manière dont les créateurs ont structuré leur contexte de réflexion et d'action.

Enfin, sur la base des constats réalisés, nous proposons, dans une dernière partie, un nouveau cadre méthodologique pour la démarche d'analyse de marché par le créateur. À cet effet, nous formulons plusieurs propositions et pistes de recherche possibles. 


\section{Analyse du marché et création d'entreprise}

Bien que le concept de marché s'inscrive dans un cadre théorique et axiomatique néoclassique, il n'existe pas de définition unique de ce terme ; cette cohabitation de plusieurs acceptions possibles contribue à brouiller notre objet d'étude. À la lecture des divers travaux disponibles, nous avons souvent l'impression que l'analyse du marché s'apparente à une notion hybride «passe-partout » susceptible de recouvrir différents aspects, du moins par des emprunts théoriques et méthodologiques partiels. Qu'entend-on exactement par analyse du marché dans le cadre précis de projets entrepreneuriaux ? S'agit-il plutôt d'une étude de l'offre et de la demande, d'une étude d'un secteur économique, d'une analyse stratégique sectorielle, d'une étude de marché ?

Aujourd'hui, l'expression " analyse du marché » apparaît trop souvent comme un énoncé commode et pédagogique pour désigner des pratiques, des approches mais aussi des réalités différentes en matière de création d'entreprise. À travers ce détour conceptuel et théorique préalable, nous avons cherché, d'une part, à mieux identifier la nature des problèmes posés par les concepts et modèles habituellement mobilisés et, d'autre part, à bien justifier et expliciter la construction de notre cadre d'analyse ainsi que le choix des dimensions retenues.

Pour tout porteur de projet, plusieurs problèmes majeurs se posent, à savoir la définition du «marché » de référence, le périmètre à retenir pour un projet donné, les niveaux d'analyse à privilégier mais aussi les dimensions à considérer.

\subsection{Par-delà la définition microéconomique... le marché est un espace construit}

Chercher à définir le concept de marché ne va pas sans poser problème. La définition qui présente le marché comme un lieu où s'échangent les offres et les demandes d'un produit idéal postule que la libre entreprise résulte d'un système capitaliste libéral, au sein duquel le fonctionnement du marché est jugé comme le seul mécanisme efficient. Nous aborderons cette question au paragraphe 2.1.1.

En réalité, les mécanismes réels et institutionnels qui président au fonctionnement de l'économie de marché (à cette économie d'échanges) laissent apparaître une très grande diversité (Marchesnay et Julien, 1996). C'est précisément autour de cette diversité que s'articulent les possibilités d'entrepreneuriat et que de nouvelles occasions d' affaires se développent, favorisant la création d'entreprise. Dès lors, il n'y aurait pas un marché, réalité ontologique, monolithique, impersonnelle, mais des marchés qui se définissent par des acteurs individuels et collectifs qui agissent, créent (Weick, 1995) et échangent. Ainsi, le marché se délimite par 
les initiatives et projets qu'engagent ces acteurs et par la représentation qu'ils se font d'eux-mêmes et des projets concurrents. Si le marché comporte une dimension objective (qui tient à la nature des produits, à la localisation des acteurs et à leurs compétences), il est aussi un espace construit par les stratégies de ces mêmes acteurs. Cette question sera traitée au paragraphe 2.1.2.

\subsubsection{Les limites de la définition microéconomique du marché}

De nombreuses recherches, notamment en stratégie et en marketing, ont souligné certaines limites d'une conception théorique néoclassique du marché (Kotler, 1986; Martinet, 1988 ; Porter, 1991 ; Kotler et Dubois, 1994).

Aujourd'hui, les chercheurs en gestion s'accordent pour reconnaître qu'il n'existe pas de définition irréfutable du marché et qu'une connaissance objective du marché est impossible. En effet, la confrontation entre l'offre et la demande ${ }^{3}$ est beaucoup plus complexe que le laisse supposer la théorie économique. L'offre et la demande n'obéissent pas à une filière unique (où l'une serait le point de départ de l'autre, l'élément manipulé), notamment du fait de la présence de différents acteurs aux dimensions psychologiques et sociologiques complexes, qui sont capables de négociations et de contre-projets. Ces agents du marché, qui ne présentent pas le comportement mécaniste de l'homo æeconomicus, sont très divers ; il y a notamment les acheteurs - le consommateur individuel ne pouvant être considéré de façon isolée - les offreurs, les distributeurs mais aussi les autres acteurs du secteur, de la filière, voire parfois des filières concurrentes. Leur intervention peut présenter de profondes différences selon la nature des marchés et des jeux possibles entre les acteurs en présence (Martinet, 1984). À ce propos, Perroux parle de «concurrence approximative » par laquelle le marchandage, la négociation, la complicité façonnent les marchés. Le marché est donc plus qu'un simple lieu d'échanges, il est aussi un lieu où les pouvoirs des différents agents s'affrontent ; il constitue un système complexe de régulation des échanges, faisant intervenir ces différents agents.

Cette évolution théorique et conceptuelle de la définition du marché est encore peu manifeste dans le champ entrepreneurial où persiste malgré tout une acception néoclassique du marché ; ainsi, il est demandé au créateur de réunir l'information fiable dont il a besoin pour définir son marché, pour s'attacher à en comprendre la structure et le fonctionnement du point de vue de la demande (le consommateur) ${ }^{4}$

3. Kotler distingue divers états de la demande (demande négative, latente, déclinante, irrégulière, pleine, excessive, indésirable).

4. Selon cette acception, le marché est défini par l'ensemble des individus qui ont acheté dans le passé une classe donnée de produit. Qu'en est-il alors des clients potentiels, notamment dans le cas d'un marché émergent? 
et du point de vue de l'offre ${ }^{5}$. En arrière-plan dominent les hypothèses de transparence du marché, d'information circulant librement et sans entraves et d'agents économiques parfaitement informés.

\subsubsection{Du marché, "champ de forces anonymes "... à un espace construit et négocié}

Depuis plusieurs années, des recherches en économie industrielle et en stratégie ayant montré que l'environnement économique n'est pas gouverné par les seuls mécanismes du marché, le champ d'investigation de l'analyse du marché a tendance à s'élargir en s'ouvrant à d'autres dimensions. Les approches d'analyse plus récentes sont moins réductrices et mutilantes pour l'environnement, qui ne se trouve plus assimilé au seul marché (Belley, Dussault et Lorrain, 1991). Le créateur est donc invité à se doter d'une vision plus élargie du marché, à tenir compte des "données du milieu » (Martinet, 1984), c'est-à-dire celles faisant référence à l'industrie, à la réglementation, à la technologie, aux évolutions culturelles, sociales, sociétales, etc. ${ }^{6}$.

Ces dernières années, les travaux successifs de Porter $(1982,1986,1991)$ ont contribué à enrichị cette réflexion sur l'analyse du marché ; on lui doit d'avoir introduit un raisonnement analytique plus complet en termes de secteur ou d'industrie plutôt que de marché (stricto sensu). Il a mis ainsi en valeur le fait que plusieurs positions compétitives peuvent être envisageables au sein d'une même industrie. La compétitivité de l'entreprise ne se trouve pas fondée sur la seule aptitude à se plier aux diktats du marché (Julien, 1994), mais sur une utilisation judicieuse des ressources engagées (notamment dans les capacités managériales), afin de déterminer l'avantage concurrentiel. Dès lors, le marché se définit plus comme un champ de forces multiples en interaction et aux limites objectivement indéfinies.

Sur la base de ces différents travaux, on remarque la diversité des périmètres et niveaux d'analyse du marché possible pour un projet de création : à savoir l'analyse de l'industrie et du secteur d'activité, l'étude des facteurs de l'environnement

5. Selon cette acception, le marché est défini comme l'ensemble des produits étroitement liés et considérés comme substituables par le consommateur, définition qui n'est pas sans poser problème.

6. À ce propos, Perroux (1973) souligne que « le marché reçoit sa forme de la société où il fonctionne et des structures sociales évolutives d'où dépendent les structures globales de production, de consommation et de répartition ; leur transformation relativement lente s'opère dans les coopérations-conflits, dans les jeux organisationnels des groupes sociaux». 
général, de la concurrence ou encore l'analyse des marchés cibles visés et de leur base géographique. Il appartient donc au créateur d'opérer un choix et de se construire ses propres frontières en fonction du projet qu'il nourrit.

\subsection{Le créateur est amené à opérer des choix...}

Dans cette perspective, l'analyse du marché constitue d'une certaine manière pour le créateur un premier engagement dans une réflexion et une démarche stratégique ; le créateur doit s'efforcer de situer son projet dans un environnement sociopoliticoéconomique «le champ d'action stratégique » (Perroux, 1973), soumis à de nombreuses influences, qui participent au processus de structuration/déstructuration du marché, au même titre que le jeu concurrentiel.

Des recherches récentes ont souligné que ce processus de construction est fortement lié au créateur, à son passé, sa formation, ses expériences antérieures, sa connaissance préalable du secteur, etc. (Cooper, Folta et Woo, 1995 ; Schwenk, 1988), mais aussi à l'idée qu'il nourrit, au projet qu'il entrevoit (Bhave, 1994) ainsi qu'à la perception initiale qu'il a de son marché.

Sur le plan méthodologique et opérationnel, ce processus de choix soulève de nombreuses questions quant à la pertinence du périmètre retenu par le créateur notamment, par exemple, pour des projets s'inscrivant sur des marchés nouveaux ou émergents.

\subsection{Des choix à opérer dans un contexte de complexité}

Parallèlement, les niveaux d'analyse du marché ont tendance à se complexifier. En effet, avec le développement de l'hypersegmentation des marchés et d'une économie de transactions, de nouveaux types de problématiques surviennent, mettant en cause la logique et les méthodes d'analyse du marché habituellement préconisées :

- en effet, le créateur fait face à un fractionnement du marché en de multiples micromarchés à durée de vie très variable. Les secteurs industriels se trouvent de plus en plus fragmentés en marchés extrêmement spécialisés, individualisés, où il est souvent difficile de se repérer, de nombreuses innovations émanant le plus souvent d'individus ou de petites entreprises s'y développant ;

- avec le développement d'une économie de transactions, le créateur a aussi à faire face à des réseaux hiérarchisés d'entreprises, où de multiples liens, relations et contrats se nouent et se dénouent, tels que les relations de sous-traitance : sous-traitance de capacité, de spécialité et également d'intelligence (Marchesnay et Julien, 1996). Parallèlement à ces entreprisesréseau se constituent aussi des réseaux plus égalitaires (réseau d'entreprises) 
de taille très semblable, travaillant sur un même site géographique ou dans une même activité ( $c f$. les districts industriels, les bassins d'emplois, les technopoles, etc.). L'espace, le territoire ne sont pas neutres ou malléables comme on le croit parfois; ils peuvent constituer un facteur essentiel dans le processus de création et de développement d'un projet, notamment pour l'accès aux ressources, l'obtention des premiers contrats.

Ainsi, cette nouvelle donnée met en lumière les limites de l'analyse microéconomique habituellement préconisée et invite le créateur à s'engager dans une analyse plus d'ordre mésoéconomique, axée sur les réseaux et sur les filières. Les travaux sur l'influence du territoire et du milieu sur l'entrepreneuriat révèlent d'ailleurs l'émergence d'espaces de transactions entre des entreprises d'une même région. Ils s'y développent des solidarités collectives, des partages de savoir, de savoir-faire dont les influences jouent sur les possibilités d'entrepreneuriat.

\section{Présentation de la démarche d'analyse et des projets étudiés}

Les développements précédents illustrent la diversité et la complexité des problèmes relatifs à l'analyse du marché pour un projet de création d'activité ; ils soulignent également la persistance mais aussi l'émergence d'interrogations majeures sur la définition du marché, du périmètre d'analyse et des dimensions à explorer.

En effet, par-delà la définition microéconomique, le marché apparaît comme un champ de forces multiples en interaction et aux limites objectivement indéfinies; le créateur d'entreprise est donc amené à opérer des choix quant au périmètre de marché qu'il va analyser, d'une part, et quant au niveau d'analyse à retenir, d'autre part, et ce, afin de se construire une représentation qui soit en adéquation avec les objectifs de son projet.

Les postulats et jeux d'hypothèses habituellement mobilisés dans les démarches actuelles sont à reconsidérer, car les frontières du marché ne sont pas données ; elles apparaissent plutôt comme des clôtures provisoires et partielles (Martinet, 1993), cognitivement et politiquement construites et décidées par le créateur, dans le cadre de son projet entrepreneurial.

Cependant, les « règles » ou pratiques actuelles d'analyse du marché ainsi que les incitations, notamment des milieux institutionnels, privilégient des démarches et perspectives beaucoup plus linéaires et objectivistes ${ }^{7}$. On peut donc s'interroger

7. C'est-à-dire des démarches comportant quatre phases séquentielles: la collecte des données, l'analyse des données, la détermination de positions et, enfin, la prise de décision. 
sur le degré d'adéquation de ces démarches, notamment en regard du caractère volontariste, téléologique et processuel que la vision du marché, comme construction, laisse entendre.

Au regard de ces problèmes et des interrogations soulevées, nous avons choisi d'articuler le cadre d'analyse autour des dimensions suivantes : le marché, le créateur et son projet en vue d'explorer les relations et interactions qu'elles entretiennent (cf. figure 1).

FIGURE 1

Cadre d'analyse initial
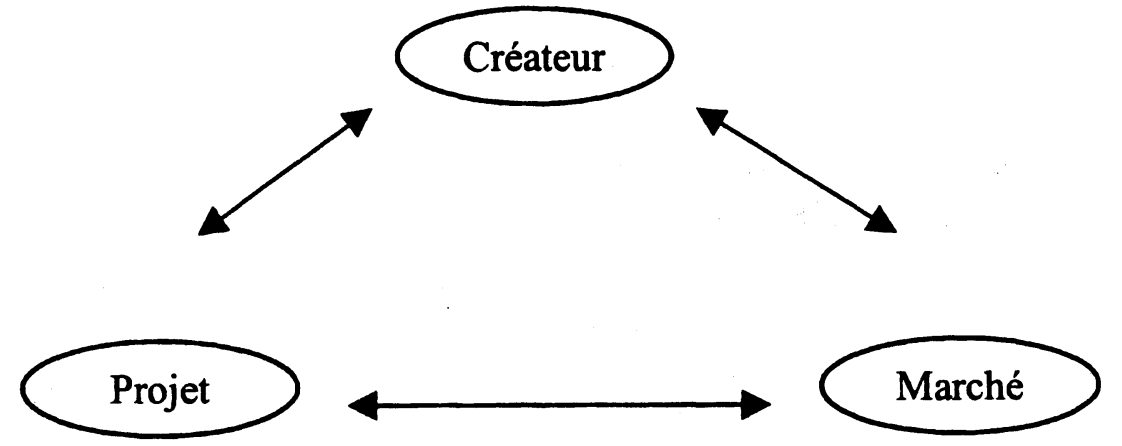

Nous avons plus particulièrement privilégié les questionnements suivants :

- À propos du marché, plusieurs problèmes se posent : l'identification du marché de référence ainsi que la définition du périmètre et des différents niveaux d'analyse de ce marché.

- Quelle vision initiale du marché le créateur privilégie-t-il naturellement ? Retient-il une vision plutôt large ou étroite du marché ?

- Selon quels processus définit-il a priori les frontières d'un marché qu'il ne connaît pas ou peu et avec lequel son projet va interagir?

En nous intéressant à la vision initiale, qualifiée aussi par Filion (1991) de vision émergente, nous cherchons à repérer autour de quels idées et concepts de prestations le créateur imagine son projet pour ensuite construire une vision centrale.

En effet, si le projet est soumis à l'influence du marché (lato sensu), il le construit et le modifie aussi par son action. Les modèles actuels ne tiennent pas suffisamment compte de cette boucle autoréférentielle et des interactions projet-marché qu'elle suscite. Quant au niveau d'analyse du 
marché, il semble se complexifier avec le développement de l'hypersegmentation des marchés et d'une économie de transactions.

- À propos du porteur et de son projet, dans la mesure où le concept d'analyse du marché est pluriel, le créateur est amené à opérer des choix, à se construire son propre périmètre, à déterminer les frontières et le « maillage » d'analyse. En nous intéressant au créateur, à sa personnalité et à ses comportements, nous avons cherché à identifier les éléments susceptibles d'avoir influencé ses processus de choix.

- Quels sont les buts du créateur?

- Quels facteurs personnels et contextuels apparaissent significatifs dans le processus de construction et d'analyse du marché, alors que le projet entrepreneurial est en train de se définir?

- Quels sont les contacts et réseaux de relations du créateur? De quelle manière les exploite-t-il ? Comment les développe-t-il ?

En effet, l'hypothèse d'un créateur, être d'une espèce rare, doté de capacités de jugement exceptionnels pour identifier son marché, apparaît invalidée au regard des études récentes menées sur les projets de créations d'entreprise (Cooper, Folta et Woo, 1995). On y découvre un entrepreneur qui, face à son projet et pour définir son marché de référence, est contraint, d'une part, par ce qu'il est et, d'autre part, par le contexte dans lequel il évolue; ainsi, l'éventail de ses choix se trouve limité par les structures socioéconomiques dans lesquelles il est inséré ainsi que par un certain nombre de facteurs, tels que sa formation, son expérience antérieure et son réseau de relations. Le créateur opère aussi ses choix sous l'influence de multiples facteurs, notamment des contraintes temporelles, informationnelles et financières. Pour définir et analyser son marché, le créateur est donc amené à composer en fonction de ces divers paramètres.

\subsection{Présentation de la méthodologie de recherche et des projets étudiés}

Ainsi que nous y invite Gartner (1989), nous avons réalisé un travail de terrain, privilégiant l'observation des entrepreneurs lorsqu'ils sont en train de créer leurs projets. Nous avons suivi la montée en puissance de projet de création, depuis le processus d'émergence du projet jusqu'au démarrage des nouvelles activités, et cela, de manière diachronique.

Cette analyse a permis l'observation et la compréhension des processus entrepreneuriaux, considérant que la création d'entreprise se définit comme un film dont le créateur est l'un des acteurs et non pas comme la photographie instantanée d'un événement (Hernandez, 1995). 


\subsubsection{Méthodologie de recherche}

Nous nous sommes attachée à étudier les porteurs de projets dans leur démarche de développement du projet d'activité : depuis l'idée originale, sa validation puis sa « mise en situation » initiale et confrontation avec les possibilités du marché. Nous avons cherché, pour chacune de ces étapes, à repérer l'approche et l'analyse du marché adoptées. Ces deux études cas ont été réalisées à partir d'observation terrain et d'analyse croisée de données recueillies auprès de différentes sources (documents écrits, entretiens avec les créateurs et des acteurs de leur environnement):

- l'observation de comportements a été réalisée par des étudiants de troisième cycle en gestion, travaillant et accompagnant les créateurs;

- pour les documents écrits retenus, l'auteure a pris en considération les descriptions initiales des projets, les plans d'affaires, des dossiers de candidature, des rapports de stage réalisés par des étudiants.

Parallèlement, l'auteure a réalisé des entretiens ouverts et semi-directifs avec les porteurs de projets et avec leur entourage proche, à fréquence régulière et sur une période de deux ans, en veillant à opérer une triangulation des données collectées par les étudiants (Yin, 1989).

- Ainsi, pour le projet 1 , la compilation et la collecte des données ont été réalisées par l'auteure ; la collecte s'est enrichie des témoignages, travaux et rapports de stage des étudiants de troisième cycle accompagnant la créatrice pendant plusieurs mois (et sur deux années consécutives). Deux autres personnes proches de la créatrice et impliquées dans le projet ont également été rencontrées. Ces entretiens ont fait l'objet de prise de notes.

- Pour le projet 2, la même démarche a été adoptée ; toutefois, à plusieurs reprises, les créateurs ont été rencontrés individuellement. Par ailleurs, une personne travaillant dans un réseau de soutien, ainsi que des acteurs de projets concurrents ont également été interviewés. Les entretiens réalisés avec les deux créateurs ont été enregistrés.

Les constats issus de l'analyse de ces données par l'auteure sont synthétisés dans les tableaux 1 et 2 .

- Le premier tableau «De l'idée au projet » présente successivement le créateur, ses buts, l'idée initiale, sa capacité de cerner et de se représenter le marché, son degré de connaissance du marché, ainsi que ses contacts et réseaux initiaux.

- Le deuxième tableau intitulé «De la perception initiale à une analyse plus construite » reprend les principales dimensions d'étude dans le processus d'analyse du marché, à savoir les pratiques et les démarches mobilisées, les réseaux développés, la mise en acte du projet (dimension d'apprentissage), la durée d'analyse et la présence d'accompagnement. 


\subsubsection{Tableaux synthétiques de présentation des projets \\ TABlEAU 1 \\ De l'idée au projet}

\begin{tabular}{|c|c|c|}
\hline & PROJET 1 & PROJET 2 \\
\hline Idée initiale & - Proposer des voyages culturels & - Développer l'usage d'Internet \\
\hline $\begin{array}{l}\text { Créateur (profil, caractéristiques, } \\
\text { expérience professionnelle initiale) }\end{array}$ & $\begin{array}{l}\text { - } 40 \text { ans, créateur expérimenté, } \\
\text { créatif, ayant une très solide } \\
\text { formation dans le domaine de } \\
\text { l'art et de l'histoire de l'art. } \\
\text { - Expérience professionnelle très } \\
\text { diversifiée dans le secteur } \\
\text { culturel. } \\
\text { - Organisation - à titre personnel } \\
\text { et de manière «bricolée »- de } \\
\text { voyages pour une clientèle } \\
\text { d'amateurs d'art. }\end{array}$ & $\begin{array}{l}\text { - Deux jeunes créateurs ( } 25 \text { ans), } \\
\text { amis de longue date, } \\
\text { respectivement diplômés d'une } \\
\text { école de gestion et d'une } \\
\text { maîtrise en informatique } \\
\text { complétée d'un troisième cycle } \\
\text { de gestion. } \\
\text { - Expérience professionnelle très } \\
\text { courte; réalisation de stages } \\
\text { dans le domaine informatique; } \\
\text { rédaction d'un mémoire sur la } \\
\text { conception et le développement } \\
\text { de serveurs Web. } \\
\text { - Familiarisation avec les } \\
\text { technologies de l'Internet et les } \\
\text { utilisations possibles } \rightarrow \text { bonne } \\
\text { compétence technique d'un } \\
\text { créateur. }\end{array}$ \\
\hline Période d'émergence de l'idée & $\begin{array}{l}\text { - Maturation lente de l'idée, au } \\
\text { fil du parcours professionnel. }\end{array}$ & $\begin{array}{l}\text { - Émergence rapide de l'idée lors } \\
\text { des stages réalisés et par la } \\
\text { pratique personnelle d'Internet. }\end{array}$ \\
\hline $\begin{array}{l}\text { Buts exprimés par le (les) } \\
\text { créateur(s) }\end{array}$ & $\begin{array}{l}\text { - Indépendance - «Vivre de sa } \\
\text { passion et gagner de l'argent } \\
\text { (en créant son propre emploi)». }\end{array}$ & $\begin{array}{l}\text { - «Être autonomes, } \\
\text { indépendants»; « développer et } \\
\text { gérer un projet dans son } \\
\text { intégralité ». }\end{array}$ \\
\hline $\begin{array}{l}\text { Situation du secteur à la période } \\
\text { d'émergence de l'idée }\end{array}$ & $\begin{array}{l}\text { - En 1994/1995, le marché du } \\
\text { tourisme culturel est en } \\
\text { développement. }\end{array}$ & $\begin{array}{l}\text { - En 1995, le développement } \\
\text { d'Internet suscite l'émergence } \\
\text { d'un nouveau secteur. }\end{array}$ \\
\hline $\begin{array}{l}\text { Capacité de cerner et de se } \\
\text { représenter le futur marché }\end{array}$ & $\begin{array}{l}\text { - Bonne capacité doublée d'une } \\
\text { certaine aisance à identifier les } \\
\text { sources d'information } \\
\text { intéressantes. } \\
\text { - Approche réaliste et pragmatique. }\end{array}$ & $\begin{array}{l}\text { - Difficultés, car le marché est } \\
\text { émergent et les créateurs sont } \\
\text { inexpérimentés. } \\
\text { - Approche visionnaire. }\end{array}$ \\
\hline $\begin{array}{l}\text { Définition du marché initial } \\
\text { (formulé par le créateur) }\end{array}$ & $\begin{array}{l}\text { - Marché du tourisme } \\
\text { - Marché des prestations culturelles }\end{array}$ & - Marché de l'Internet \\
\hline $\begin{array}{l}\text { Degré de connaissance initiale du } \\
\text { marché }\end{array}$ & - Bonne connaissance globale. & $\begin{array}{l}\text { - Bonne connaissance technique } \\
\text { mais insuffisante des } \\
\text { dimensions socioéconomiques. } \\
\text { - Beaucoup d'interrogations et } \\
\text { d'incertitudes sur les aspects } \\
\text { techniques. } \\
\text { - Faible connaissance des } \\
\text { secteurs proches (informatique, } \\
\text { télécommunications). }\end{array}$ \\
\hline $\begin{array}{l}\text { Contacts initiaux, réseau de } \\
\text { relations du créateur }\end{array}$ & $\begin{array}{l}\text { Contacts nombreux dans le secteur } \\
\text { de la culture; réseau de relations très } \\
\text { actif dans le domaine de la } \\
\text { médiation culturelle. }\end{array}$ & $\begin{array}{l}\text { Peu de contacts et réseau peu } \\
\text { développé (hormis les contacts } \\
\text { établis lors des stages). }\end{array}$ \\
\hline $\begin{array}{l}\text { Vision centrale : la représentation } \\
\text { du projet dans son marché (exprimé } \\
\text { par le créateur en ses termes) }\end{array}$ & $\begin{array}{l}\text { «Développer une offre de voyages } \\
\text { culturels pointus auprès d'une } \\
\text { clientèle de particuliers. » }\end{array}$ & $\begin{array}{l}\text { «Développer une offre de } \\
\text { connection et de services autour } \\
\text { d'Internet, auprès du grand public et } \\
\text { des entreprises. » }\end{array}$ \\
\hline
\end{tabular}




\section{TABLEAU 2}

\section{De la perception initiale à une analyse plus construite du marché}

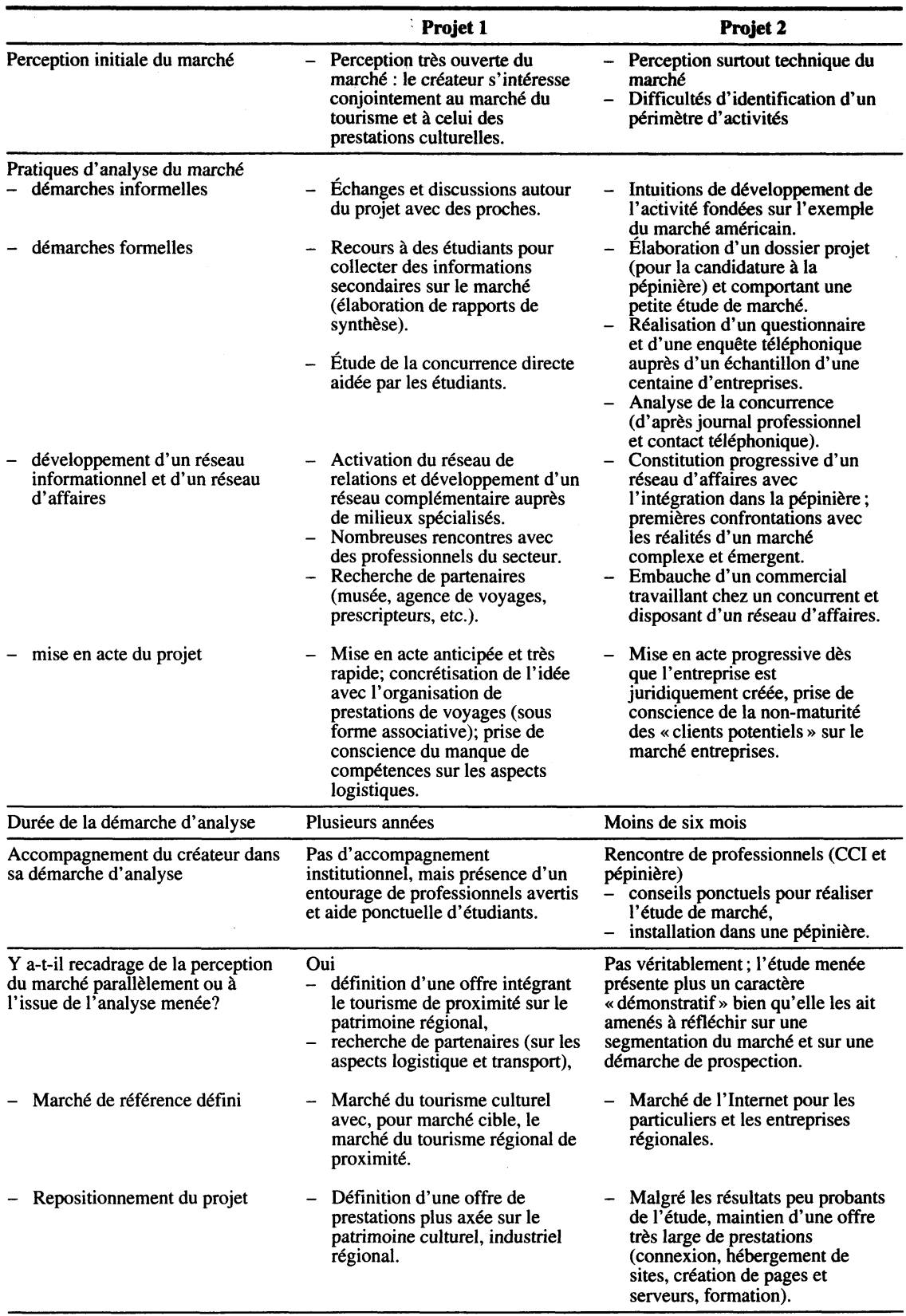




\section{Interprétation proposée}

La démarche de travail adoptée a permis, à partir de l'observation et de l'analyse des données, d'inférer certaines remarques, de relever des divergences ou des similitudes entre les situations concrètes et les modèles ou méthodes couramment mobilisés.

Il ressort de ce travail que les démarches adoptées par les deux créateurs ne suivent pas à la lettre la méthodologie habituellement préconisée. Plutôt que de souligner certaines maladresses ou « erreurs » commises et de formuler une critique de leur démarche au regard des critères habituels, nous avons cherché à tirer des enseignements en nous intéressant à ce que font effectivement les entrepreneurs lorsqu'ils analysent le marché, tout en ayant bien conscience du caractère contingent des comportements et démarches observés.

La comparaison des situations d'analyse du marché relatifs à ces deux projets met en lumière des phénomènes suivants :

- si, initialement, les créateurs ont plutôt privilégié une définition a priori du marché (4.1.), nous avons constaté le caractère itératif, processuel et expérimental d'élaboration de cette vision et de cette connaissance du marché (4.2.1.). Dans ce processus, les réseaux de relations tissées par les créateurs (4.2.2.) ainsi que les premières concrétisations du projet ont revêtu un rôle crucial (4.2.3.);

- par ailleurs, les créateurs ont éprouvé des difficultés à faire articuler et à faire évoluer de façon cohérente leur vision du projet avec celle de leur marché et de leur environnement (4.3.).

En préambule, il faut souligner que la terminologie employée par les créateurs ou les autres acteurs du milieu entrepreneurial (pépinière, société de capital risque) est peu stabilisée; les concepts de marché, secteur et environnement manquent cruellement de clarté.

\subsection{Une définition a priori du marché}

Le problème de la définition du marché de référence (selon une acception large) n'a jamais été précisément abordé et même exprimé par les créateurs : ils se sont plus appuyés sur une définition a priori du marché (« marché du tourisme et marché des prestations culturelles », «marché de l'Internet»), qui a émergé spontanément, sous l'influence respective des expériences professionnelles, des rencontres et collectes d'informations. Très rapidement, les créateurs développent une conscience assez aiguë de l'activité nouvelle et de son marché de référence. La perception du marché semble s'amorcer dès l'émergence de l'idée (parfois antérieurement), sans aucun questionnement préalable. 
Cette définition a priori présente des conséquences sur la perception du périmètre d'analyse du marché. Si le créateur $\mathrm{A}$ a très tôt adopté une vision large de son marché où il va repérer différents sous-systèmes interagissant (culturel, touristique, logistique, etc.), les créateurs du projet $B$ privilégient une vision plus étroite de leur marché dans un contexte global, imprécis et assez flou, concernant Internet, ses technologies et ses usages.

En effet, dès l'émergence de l'idée initiale, le créateur $\mathrm{A}$ a déjà conscience que ce projet s'inscrit dans un univers s'articulant autour du marché du tourisme et du marché des prestations culturelles. Il va s'intéresser à ces deux marchés en parallèle, d'autant plus qu'à l'époque, peu d'informations sont disponibles sur le marché spécifique du tourisme culturel ; pour ce faire, il se fait aider dans cet effort d'analyse par des étudiants, qui rechercheront des données secondaires auprès d'organismes tels que l'Institut national des statistiques et des études économiques, la Direction régionale aux affaires culturelles, le Comité régional du tourisme et le ministère du Tourisme. Cette perception initiale du marché permet au créateur de respecter l'extrême diversité et complexité du marché, tant au niveau de l'offre que de la demande, et de s'intéresser à la présence de nombreux acteurs aux profils très disparates. La curiosité naturelle du créateur, sa bonne connaissance des profils des clients potentiels l'incitent à toujours pousser plus loin ses investigations («à en savoir toujours plus »), ce qui lui permet de maintenir une vision très large du périmètre d'analyse du marché ; il raisonne plus en termes de secteur, sans toutefois l'exprimer dans ces termes, et sait bien discerner les interactions entre les différents acteurs impliqués plus ou moins directement dans son projet.

Pour les créateurs du projet $B$, la définition du marché de référence est à l'origine beaucoup plus implicite; la question de l'identification du marché de référence et du périmètre n'est pas explicitement exprimée, notamment liée au caractère turbulent et complexe de ce marché émergent. De toute évidence, il s'agit pour eux du marché de l'Internet, sans qu'ils s'interrogent sur la réalité qu'il recouvre. Le créateur de formation technique déclare avoir eu tôt l'intime conviction que le marché visé était le marché de l'Internet et que ce dernier allait considérablement se développer au regard des situations des pays étrangers et du retard de la France (en 1994) dans ce domaine. À cet égard, les créateurs ont été perturbés par le manque d'informations claires et précises ( « une information presse trop générale et souvent contradictoire ») sur ce marché très émergent. Ils disposent également de peu de données factuelles pertinentes sur l'environnement global, Internet étant en France un phénomène nouveau; ils vont procéder à une mise en forme de cet environnement, ce que Weick (1969) qualifie d'enactment, en se construisant leur propre cadre d'interprétation et de significations communes.

Ils vont privilégier une vision étriquée du marché axée sur la dimension technique, puis ils vont très vite se centrer uniquement sur le marché cible, c'est-àdire l'analyse d'une demande possible. Ils s'interrogent peu sur les dimensions 
économique, politique, sociale, etc., de l'environnement du projet, ainsi que sur les acteurs présents et/ou possibles (fournisseurs, concurrents potentiels, etc.). Quelques mois après la création, ils découvrent que cette vision trop étroite du marché leur pose des problèmes, étant donné les fortes imbrications de ce marché avec celui des télécommunications et de l'informatique et l'influence respective qu'exercent des acteurs de profil et de taille très différents, comme France Telecom avec Wanadoo, Microsoft avec son offensive tardive sur l'Internet, capables de développer des stratégies d'arène (Brechet, 1996).

\subsection{Le caractère processuel, itératif et expérimental de l'analyse du marché}

Si l'on considère que tout projet entrepreneurial transite par plusieurs phases, à savoir le prédémarrage (ou la gestation de l'idée), le démarrage puis le postdémarrage et la consolidation (Marchesnay et Julien, 1996), il est communément admis que l'analyse du marché doit s'inscrire dans la première étape.

Au regard des observations réalisées, il nous semble souhaitable de revenir sur cette idée dominante. L'analyse du marché ne constitue pas une étape spécifique - à mettre entre parenthèses - à isoler dans le temps. Bien au contraire, pour les deux cas, elle s'est inscrite dans un processus global d'approche du marché ayant débuté antérieurement (parfois conjointement avec l'émergence de l'idée originale) et elle s'est poursuivie avec la mise en acte du projet dans un marché en constante évolution et avec lequel chaque projet a interagi.

L'élaboration d'une vision du marché ainsi que la démarche d'analyse adoptée présentent un caractère processuel et itératif (4.2.1.) dans lequel les réseaux de relations tissés par les créateurs (4.2.2.) et les premières concrétisations du projet ont joué un rôle crucial (4.2.3.).

\subsubsection{Une approche itérative et procédurale du marché}

L'étude du terrain révèle des pratiques fort éloignées des perspectives habituelles, au caractère très linéaire et objectiviste, à savoir collecte de données, analyse des données, détermination de positions puis, enfin, prise de décision. Implicitement, elles postulent que d'entrée de jeu les problèmes sont bien posés et identifiés et qu'il appartient au créateur de les résoudre en procédant par étapes successives, l'analyse faisant émerger des réponses, susceptibles d'alimenter des processus de décisions rationnels.

Les observations des deux projets ont mis en lumière des démarches d'analyse plus procédurales autour de processus dialogiques et itératifs, selon un cheminement que l'on peut qualifier d'hélicoïdal. Pour chaque projet étudié, les créateurs n'ont pas eu d'emblée recours à une démarche délibérée articulée autour d'une logique 
clairement et formellement établie a priori ; ils ont préféré procéder par investigation ponctuelle, «à petits pas », par ajustement progressif de l'idée, au gré de leurs intuitions, rencontres, échanges d'information, analyses mais aussi problèmes rencontrés. Cette approche incrémentale du marché, par imprégnation progressive est très éloignée de l'image médiatisée d'une conception mécaniste, programmatique du processus d'analyse de marché, où les créateurs s'engagent dans une recherche systématique et organisée d'informations.

Si les créateurs B ont plus privilégié un travail de « détective» cherchant ici et là à capter des données composites, le créateur A s'est efforcé pendant plusieurs années de « remonter des filières » à partir de nombreuses rencontres et entretiens effectués, dans le but de collecter des éléments d'information, mais aussi de se rassurer sur la validité de son idée. Progressivement, il s'est construit une cartographie du territoire du tourisme culturel - à partir d'éléments topographiques relevés ici et là - recomposant en quelque sorte une forme de puzzle sans modèle original. Le créateur $\mathrm{A}$ a ainsi privilégié des données qualitatives sur les acteurs locaux, les pratiques commerciales, les relais, les pôles d'influence, les organisations prescriptrices, etc., qu'il a interprétées après échanges préalables avec des tierces personnes.

Dans ce processus d'analyse itératif, plusieurs dimensions nous sont apparues significatives : l'existence et l'influence exercée par les réseaux du créateur (4.2.2.), la mise en acte du projet et les apprentissages qui en résultent (4.2.3.).

\subsubsection{L'influence décisive des réseaux}

L'étude des deux projets révèle l'influence majeure des relations et réseaux de contacts du créateur sur la connaissance du marché. L'intensité de cette dimension relationnelle a permis aux créateurs, d'une part, d'avoir accès à des informations utiles, d'autre part, d'engager un dialogue riche et exploratoire auprès d'interlocuteurs variés ${ }^{8}$; elle a pu inciter les créateurs à envisager des partenariats possibles, parfois très en amont de la phase de démarrage du projet. Ces réseaux de relations ont constitué un facteur décisif dans la prise de conscience des créateurs sur le tissu d'acteurs agissant sur le marché.

Le créateur A a cherché, dans un premier temps, à se positionner au sein d'un large réseau de relations environnementales - relations avec les organisations publiques et parapubliques, avec les clients, avec les fournisseurs, avec les prescripteurs, etc. Il a consacré beaucoup de temps à travailler ce réseau de contacts et d'influences. Cette position lui a permis de repérer dans cet environnement quelles

8. Par ces rencontres, le porteur du projet est amené à échanger autour de problèmes complexes, non structurés et à reconsidérer certaines de ses conceptions et visions initiales. 
étaient les sources d'occasions d'affaires : selon le vieil adage, "ce que vous connaissez est bien moins utile que les personnes que vous connaissez ». Ce créateur a ainsi découvert l'importance des partenariats, amorcés tout d'abord avec le musée des Beaux-Arts, puis avec l'agence de voyages; il a également pris conscience que les transactions de nature commerciale s'accompagnent aussi de transactions non marchandes (Perroux, 1973) et qu'il est important de savoir faire usage de ce pouvoir sur le marché mais aussi hors du marché (notamment par l'accès à des informations particulières (signaux faibles, information d'anticipation) ou encore par le développement de relations privilégiées auprès de prescripteurs ciblés... Multiplier les rencontres est apparu très salutaire pour le projet $A$ et son inscription future dans un réseau d'affaires. Ce frayage relationnel (Koenig, 1994) suppose une forte dépense d'énergie, mais il a permis au créateur de se doter de relations actives pour le développement de son projet et pour nouer des partenariats décisifs.

Les porteurs du projet $\mathrm{B}$ n'ont à l'origine pas privilégié cette dimension relationnelle dans leur approche et analyse de leur marché. La réalisation de leurs premiers contrats leur a fait prendre conscience de la faiblesse de ce réseau de contacts et de relations, aussi bien auprès des fournisseurs que des clients ou prescripteurs éventuels. Leur insertion territoriale dans la pépinière d'entreprises les a toutefois aidés à compenser cette faiblesse initiale du projet ainsi que l'embauche d'un commercial venant d'une entreprise concurrente (et ceci, deux mois après la création de l'entreprise).

Nous avons constaté que la constitution et le développement de ces réseaux d'informations et d'affaires ont permis aux trois créateurs de se construire un «espace de transactions » (Julien et Marchesnay, 1996) pour leurs projets ; cet espace a contribué à une certaine définition, voire à la stabilisation du marché et de l'environnement global du projet. Le fait que l'édification de ces réseaux soit apparue tôt dans le processus d'analyse du marché semble favoriser un plus grand degré d'ouverture et une meilleure compréhension des phénomènes environnementaux et des changements qui s'y opèrent (évolution du marché, de la concurrence, etc.).

\subsubsection{La mise en acte, une étape décisive dans l'analyse du marché}

Parallèlement à une connaissance théorique, celle habituellement évoquée dans les modèles dominants, nous avons constaté pour les deux projets que les premières «mises en acte » constituent de réels leviers dans la connaissance pratique du marché. L'engagement dans l'action avec les premiers contrats ou prestations a réellement permis d'évaluer certaines caractéristiques du secteur d'activité pénétré. Cette mise en acte du projet a notamment fait découvrir aux créateurs B des caractéristiques très tangibles du marché pénétré (complexité, turbulence, accessibilité) et le fort degré de dépendance à l'égard des fournisseurs (de modems, d'accès aux 
réseaux de télécommunication, etc.); ils ont découvert certaines règles du jeu concurrentiel et le degré d'hostilité et d'agressivité des concurrents locaux (en quelques mois, apparition de cinq nouveaux concurrents sur le même espace de marché régional).

Sur ce point, le créateur A qui avait engagé une mise en acte très anticipée, par l'organisation « bricolée » de voyages culturels, s'était déjà infiltré dans l'espace de transactions marchandes et non marchandes et avait provoqué une confrontation plus rapide du projet à l'inertie de l'environnement. Il a ainsi pris conscience de questions cruciales : comment trouver des clients ? Comment développer des circuits de commercialisation ? Comment convaincre des fournisseurs et prescripteurs d'un partenariat?

En donnant corps et surtout vie au projet, en accédant au champ de la compétition, les créateurs entrepreneurs ont découvert des dimensions plus effectives de leur univers socioéconomique et de leurs marchés cibles. Ils ont mis ainsi en lumière des éléments de vulnérabilité de leur projet, des difficultés nouvelles, des obstacles mal évalués (tels que la rivalité des concurrents, $c f$. projet B). Ils ont pu ainsi mieux cerner certaines compétences distinctives et identifier leurs possibilités de démarquage par rapport aux offres concurrentes. Les créateurs sont devenus plus circonspects quant aux informations fournies par leur vision initiale et leur connaissance « théorique » du marché. Les problèmes gagnant à la fois en acuité et en intensité, ils ont relativisé un certain nombre de données préalables, car le marché devenait plus palpable. Cette mise en acte du projet a aussi permis une familiarisation avec des dimensions plus contingentes, telles que les relations préexistantes entre acteurs, les spécificités «tacites » du métier et les conventions dominantes.

\subsection{La difficulté de formaliser la connaissance du marché au sein d'une vision systémique du projet}

Par ailleurs, nous avons constaté que les créateurs ont pris conscience du foisonnement des dimensions d'analyse à considérer et leur fort degré d'imbrication les a parfois gênés, voire découragés à poursuivre leur investigation plus en profondeur. Cela se traduit par de réelles difficultés d'organisation, de structuration des données dont ils disposent. Les impressions de désordre, de confusion, de « préoccupations et problèmes partant tous azimuts » sont vécues différemment selon les créateurs étudiés.

Si le créateur A a assez bien géré cette difficulté, notamment en raison de ses qualités personnelles et de son réseau de relations avec lequel il a beaucoup discuté, les créateurs $B$ ont voulu très tôt introduire des éléments d'ordre, de stabilité, de certitude autour du projet, ce qui les a parfois amenés à évacuer ou à minimiser certains problèmes dans leur analyse du marché cible. Ils se sont engagés très tôt 
dans une démarche très analytique (répondant aussi à certaines obligations de la candidature à la pépinière), qui s'est articulée autour d'une "petite étude de marché ». Ce travail $\mathrm{d}$ 'analyse a abouti à un document écrit très édulcoré où les raisonnements sont très (trop) démonstratifs; l'incertitude, la méconnaissance, le doute, voire le paradoxe semblent évacués ; ainsi, leur étude fait ressortir un taux élevé de méconnaissance de l'Internet et de ses usages dans les petites entreprises interrogées. Les créateurs cherchent plus à raisonner en termes de taux de croissance, de chiffre d'affaires, de parts de marché visé (peut-être en vertu d'un certain conformisme), en faisant référence notamment à des données très macroéconomiques. Ils privilégient une description statique, négligeant les influences des interactions entre l'environnement et le projet. Pour les créateurs B, il y a une perte de variété entre l'analyse du marché exprimée oralement, notamment lors des interviews, et sa transcription dans le dossier projet destiné à la commission d'admission de la pépinière.

Aussi bien pour le projet A que pour le projet B, la modélisation de l'univers d'action du projet pose problème; la compréhension des interactions entre les différentes composantes du système projet environnement et créateur, apparaît délicate ; le repérage des différentes interactions (voire rétroactions) entre le projet et les sous-systèmes externes, l'influence positive et négative de ces interactions sur la réalisation du projet, l'impact des facteurs de l'environnement général sur le projet sont des questions délaissées au profit de présentations plus souvent statiques et descriptives.

\section{Propositions autour d'un nouveau cadre méthodologique}

Les projets étudiés témoignent de pratiques d'analyse de marché encore trop insuffisamment étudiées et révèlent notre incapacité à bien comprendre les processus à l'œuvre. À partir des constats précédents, il nous semble souhaitable que la mise en cause de l'analyse du marché comme facteur explicatif possible dans les défaillances des jeunes entreprises soit reconsidérée. À ce titre, nous avons cherché à proposer un nouveau cadre méthodologique pour la démarche d'analyse de marché, par le créateur (5.1.); cela nous amène à formuler des propositions sur les spécificités et conditions d'efficacité d'une analyse de marché dans le cadre de projets entrepreneuriaux (5.2.).

\subsection{Un nouveau cadre méthodologique pour l'analyse de marché}

Dans la mesure où l'analyse du marché revêt une forte valeur d'enjeu aussi bien pour le créateur que pour les acteurs et professionnels du milieu entrepreneurial, il nous apparaît souhaitable d'aborder l'analyse du marché comme la pierre angulaire 
d'une première démarche de réflexion stratégique que le créateur engage sur son projet d'entreprendre (Brechet, 1996). À cet effet, nous sommes partie du modèle d'analyse stratégique proposé par M. Marchesnay (1993), afin de proposer un nouveau cadre méthodologique d'analyse du marché. Ce cadre s'inscrit dans un système global structuré autour de quatre composantes en interaction permanente : l'une concerne le créateur (son profil, son expérience professionnelle, son expérience et connaissance du marché et du métier, ses réseaux), une autre concerne les dimensions et caractéristiques progressives du projet (d'une part, l'offre - c'est-àdire les activités et prestations retenues, et d'autre part, le système d'offre, c'est-à-dire la configuration managériale et organisationnelle relative au projet) et la dernière porte sur les différents systèmes environnementaux, en particulier technique, social, économique et politique.

FIGURE 2

\section{Un cadre systémique d'analyse}

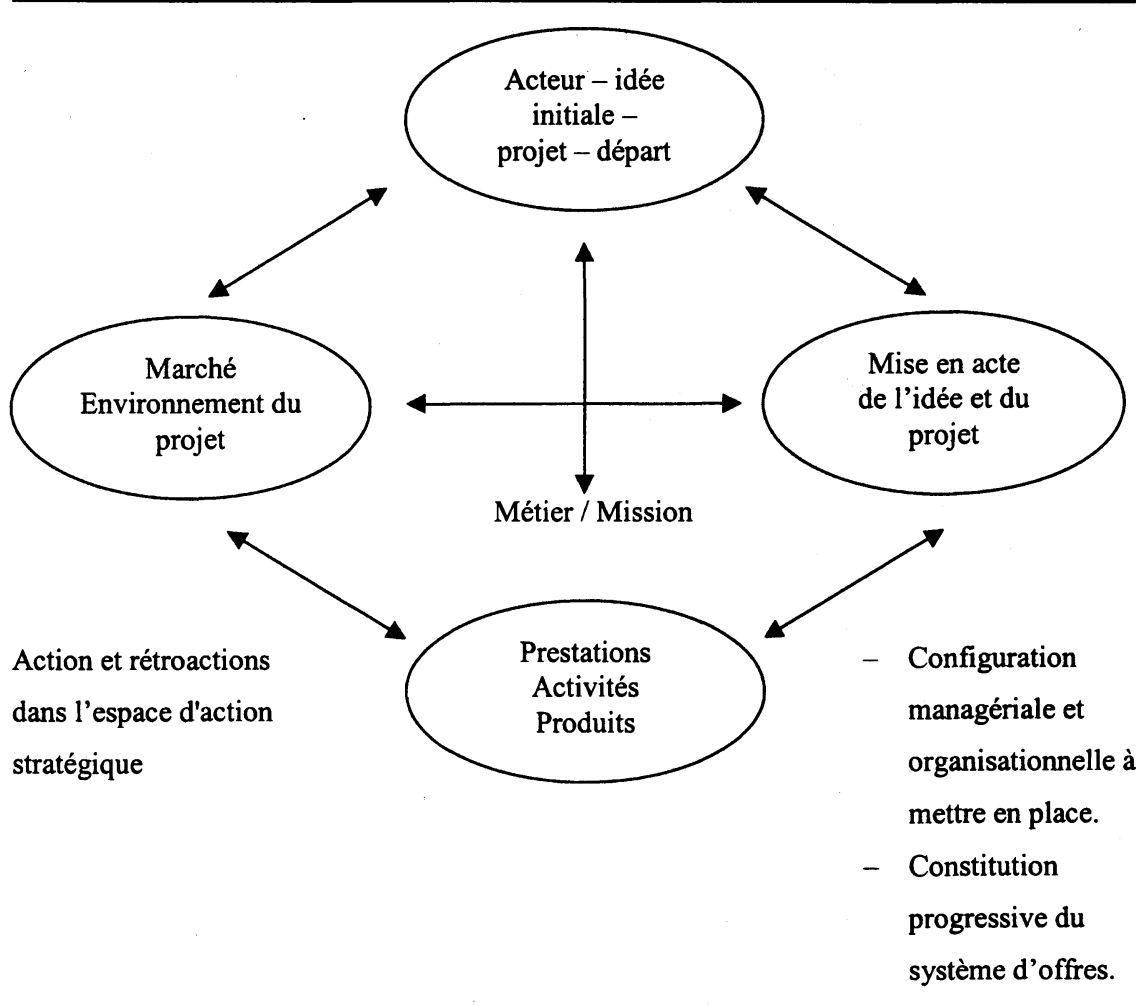


En adoptant ce cadre d'analyse, le créateur peut, par une lecture systémique du projet dès la phase de prédémarrage, être mieux armé pour réfléchir et agir sur le développement de son projet; il peut ainsi mieux tenir compte des relations et interactions du projet avec ces différents sous-systèmes et veiller à la cohérence entre ces diverses variables.

Ce nouveau cadre méthodologique peut fournir plusieurs atouts :

- il donne au créateur une meilleure visibilité des différents axes d'élaboration de sa vision du marché, ainsi que des différents processus concernés (par exemple, le processus de collecte d'information sur l'environnement et celui de mise en acte de l'idée);

- il permet également au créateur de mieux prendre conscience de la forte interaction des dimensions entre elles; ce dernier peut ainsi opérer des bouclages en arrière, notamment sur l'idée initiale et sur la validation de l'idée ; il peut recadrer et redéfinir son projet (notamment le couple métier/ mission), ce qui d'emblée aura des incidences sur la question de l'identification du marché de référence, du périmètre d'analyse, des frontières du projet et de la relation entre le positionnement concurrentiel recherché et l'avantage concurrentiel à bâtir (choix de l'offre et du système d'offre);

- ce cadre d'analyse présente aussi l'intérêt d'être à géométrie variable et de pouvoir évoluer selon le niveau d'avancement et de maturité du projet. Il permet également de tenir compte d'évolutions au caractère plus erratique, de changements émergents susceptibles de survenir à chaque pôle.

\subsection{Propositions}

Parallèlement à ce cadre d'analyse, il nous apparaît important d'introduire plusieurs propositions complémentaires sur l'analyse de marché dans le cadre de projets entrepreneuriaux :

- l'analyse du marché doit se définir comme un objet de représentation polymorphe et dynamique ;

- l'analyse du marché doit être un outil servant à développer l'intelligence des problèmes sur l'espace d'action stratégique du projet ;

- l'analyse du marché doit permettre de guider les processus d'apprentissage du créateur analysant son marché. 


\subsubsection{L'analyse du marché, objet de représentation polymorphe et dynamique}

L'analyse du marché constitue un objet de représentation polymorphe et dynamique, pouvant s'exprimer sous des formes diverses et variées, selon le profil du créateur, la nature de son projet et le marché concerné. L'unicité terminologique ne doit donc pas nous abuser et nous laisser croire à un objet unique et stable, reposant sur une démarche standard, privilégiant une connaissance statique et rétrospective, déclinable quel que soit le contexte. Dans la mesure où l'analyse de marché constitue un domaine d'investigation extrêmement complexe, où surviennent de manière incessante des discontinuités, en raison notamment de l'évolution rapide des besoins, de leur fractionnement et de l'apparition de nouveaux acteurs et projets, il semble préférable de chercher à promouvoir un cadre ouvert de réflexion et d'action, s'articulant autour de ces quatre pôles en interaction. Le créateur peut ainsi veiller à la mise en relation et en cohérence de ces composantes ( $c f$. figure 2).

Dans cette perspective, l'objectif de l'analyse du marché devient double: permettre au créateur d'établir, à un instant donné, une carte stable d'un territoire en agitation permanente, tout en faisant en sorte que cette représentation soit évolutive et dynamique. $\mathrm{Si}$, initialement, la représentation du champ de la réflexion se limitait aux seuls couples produits-marchés, aujourd'hui, elle doit s'enrichir de représentations de la situation environnementale et des interactions entre le projet et ses contextes sociotechnique et politico-économique, soit l'espace d'action stratégique. Cette conception suppose que l'analyse du marché ne se limite pas à la seule connaissance des transactions marchandes que le créateur souhaite développer sur le marché avec les acteurs proches, en particulier l'acheteur, le distributeur, le fournisseur; elle doit aussi porter sur la connaissance des autres agents et des réseaux de relations, interférant dans ces transactions, car ils contribuent aussi à la structuration du marché.

\subsubsection{L'analyse du marché pour développer l'intelligence des problèmes et du champ des possibles}

En privilégiant un cadre méthodologique plus dynamique et processuel (cf. figure 2), l'analyse du marché ne se limite pas à une réflexion quasi mécanique, pur exercice de style, destiné à cautionner la validité d'un projet dans le cadre d'un plan d'affaires ; elle vise plutôt à amener le créateur à s'interroger sur le marché de référence qu'il s'est défini a priori, en essayant d'y repérer la dimension objective et la dimension construite. Pour parvenir à dresser à un instant $\mathrm{T}$ une certaine cartographie du territoire de réflexion et d'action de son projet, le créateur doit chercher à se renseigner sur une filière, sur un secteur d'activité, bref, à s'en imprégner, afin d'y découvrir des champs de problèmes sur l'espace d'action stratégique de son projet; il doit s'efforcer de structurer ces espaces de problèmes à 
partir de données générales souvent floues, imprécises et parfois même contradictoires qu'il aura réunies selon des processus très divers : enquêtes, interviews, analyses officielles, réseaux, mise en acte, etc.

L'analyse du marché dépasse alors l'étude statique et descriptive d'évolutions passées, de situations entérinées et se définit plus comme une représentation riche et complexe d'une situation mouvante, incertaine offrant au créateur des repères, des éléments utiles pour l'identification de problèmes actuels et potentiels concernant son offre et la constitution de son système d'offres.

L'objectif de cette cartographie n'est donc pas de reproduire une réalité hypothétique mais de favoriser chez le créateur des situations d'interrogation stratégique, de doute et de questionnement, à la fois sur la définition du marché, les frontières et les dimensions retenues. Les réflexions et analyses engagées doivent plus être considérées comme des pistes possibles pour améliorer l'intelligence que le créateur a de la situation et/ou pour stimuler sa réflexion et son imagination. Dans un premier temps, le créateur est invité à développer une intelligence du champ des possibles, à s'interroger au préalable sur ses propres perceptions du marché, notamment autour des thèmes suivants : quelle connaissance initiale a-t-il de ce marché ? Comment s'est-elle constituée ? Comment définit-il le marché (structure, activités, acteurs dominants, etc.) ? Quels critères de segmentation retient-il ? A-t-il discuté de sa perception du marché avec d'autres individus? A-t-il rencontré des experts, des professionnels du secteur ? Comment le projet se place-t-il sur ce marché ?

Cette étape préalable de questionnement nous semble indispensable avant d'envisager la nature et l'intensité des actions à entreprendre par le créateur pour agir sur les différents marchés de l'amont, fournisseurs, financiers, personnel, etc., et de l'aval, consommateurs, distributeurs, prescripteurs. Comment envisage-t-il le passage à l'acte (contacts clients, contacts commerciaux, précarnet de commande, etc.) ? Quels efforts le créateur va-t-il déployer pour agir sur cet environnement et permettre à l'entreprise d'atteindre les performances souhaitées ? Quels sont les moyens à mettre en œuvre au sein du système d'offre?

Par cette approche plus dynamique et réaliste, le créateur peut mieux envisager la nature des rétroactions environnement-projet et évaluer leurs incidences sur les différents scénarios du plan d'affaires.

\subsubsection{L'analyse du marché, pour guider les processus d'apprentissage du créateur}

Sur la base des remarques précédentes, l'analyse de marché n'a donc pas comme objectif de permettre une modélisation détaillée de la réalité perçue, mais devient l'esquisse d'une cible susceptible de guider les processus d' apprentissage du créateur. En effet, la connaissance du marché semble s'acquérir autant dans le développement 
d'un savoir que par l'acquisition d'un savoir-faire dans l'action (Koenig, 1994). Si la connaissance «théorique » permet de préciser l'idée, de cerner le marché de référence, de repérer ses caractéristiques et évolutions possibles ainsi que des espaces de problème, le créateur se trouve - lors de la phase de démarrage, qualifiée aussi de montée en compétitivité - réellement confronté au marché ; ce dernier va commencer à imposer ses contraintes, à travers les premières commandes clients, les réactions des concurrents, les échanges et transactions marchandes et non marchandes, etc.

Cette étape apparaît cruciale pour le développement du projet d'entreprendre, car le créateur vit réellement le marché qu'il a auparavant « enacté » (Weick, 1969). Au cours de cette phase qui peut être riche d'apprentissages, le créateur doit savoir prendre le temps de modifier sa perception du marché (double loop learning ; Argyris, 1995). Face à des situations dynamiques, changeantes et complexes, le créateur doit s'attacher à analyser les actions en cours et à intégrer le rôle qu'elles ont joué dans la perception et la connaissance du marché. Ainsi, certains événements peuvent amener le créateur à redéfinir l'activité, à repenser son offre, son système d'offres, sa démarche commerciale, etc.

On peut regretter qu'actuellement on ne tienne pas suffisamment compte de ces processus d'apprentissage dans le cadre d'une démarche de création. Leur meilleure connaissance pourrait enrichir la problématique étudiée et permettre aussi l'évolution de l'exercice du métier d'entrepreneur.

\section{Conclusion}

À l'aide des apports de la littérature et des premiers éléments d'une étude de cas, nous avons constaté que ce domaine de l'analyse de marché dans le cadre de projets entrepreneuriaux est encore mal connu et insuffisamment traité. Les idées et pistes de réflexion formulées demandent à être approfondies par des travaux complémentaires pour bien tester la validité de notre cadre méthodologique et des propositions qui s'y rapportent. Nous pourrions ainsi être en mesure de présenter aux créateurs des cadres de réflexion et d'action plus réalistes et adaptés à leurs préoccupations.

Toutefois, dans la mesure où le créateur sera toujours soumis à des contraintes fortes pour réaliser son analyse du marché, notamment le manque de temps et de moyens qui souvent aboutissent à une analyse et une réflexion abrégée, il nous semble important d'envisager de nouveaux dispositifs d'accompagnement du créateur, qui soient plus présents au fil de l'évolution du projet. On attend de ces dispositifs qu'ils permettent au créateur une meilleure insertion au sein de réseaux, qu'ils l'aident à anticiper la mise en acte du projet et surtout qu'ils le guident dans les processus d'apprentissage lors des phases de prédémarrage et de démarrage du projet. Voici autant de pistes à suivre afin que le créateur développe dans une plus grande efficacité son analyse du marché. 
De telles propositions nécessitent une révision des outils et modèles utilisés ainsi que des modes actuels d'accompagnement des créateurs, qui devraient plus intervenir après la création, afin d'apporter aux créateurs un soutien actif dans leur apprentissage du marché. Trop souvent, les dispositifs actuels d'aide et d'accompagnement s'arrêtent à la conception et au montage du projet, avec l'hypothétique validation de l'existence d'un marché possible et potentiel; or, les informations réellement pertinentes surgissent lors de la mise en œuvre du projet, lors de la rencontre et/ou confrontation directe avec une réalité de premier ordre, au cours de laquelle l'entrepreneur apprend son marché dans une sorte d'école de l'erreur. Pour réaliser ces apprentissages, le créateur est souvent assez seul, démuni et submergé par les problèmes opérationnels relatifs à son projet d'entreprendre. Plongés dans un environnement de plus en plus complexe et turbulent, les créateurs sont amenés à faire des apprentissage en « marchant ». Ainsi, pour certains marchés nouveaux ou émergents, ou pour des projets inédits, accepter d'apprendre l'essentiel sur le marché et ce qu'il convient de faire dans le déroulement même de l'action peut perturber.

Dans ce sens, on peut considérer que l'analyse du marché est inséparable de l'expérience que le créateur y acquiert, c'est pourquoi il nous semble vivement souhaitable que soient mises en place de nouvelles formes d'accompagnement et de soutien des créateurs, notamment en focalisant sur les premières années du développement du projet productif.

\section{Bibliographie}

ARGYRIS, C. (1995), Savoir agir, Paris, InterÉditions.

AVENIER, M.J. (1989), «L'organisation d'un objet multidisciplinaire à un projet transdisciplinaire complexe», Document de recherche, Université Aix-en-Provence.

BhAVE, P.M. (1994), «Process model of entrepreneurial creation», Journal of Business Venturing, vol. $9, \mathrm{n}^{\circ} 3$, mai.

Belley, A., L. DUSSAULt et J. LORRAIN (1991), «Le plan d'affaires», Revue Organisation.

BLOCK Z. et I.C. MACMILLAN (1985), «Milestones for successful venture planning》, Harvard Business Review, p. 184-196.

BRECHET, J.P. (1996), Gestion stratégique : Le développement du projet d'entreprendre, Paris, Eska.

COLLECTIF, FinANCE CONSEIL (1995), «Le guide de la création d'entreprise », Business Plan, de la réflexion à l'action, Paris, Finance Conseil.

COOPER, A.C., T.B. FOLTA et C. Woo (1995), «Entrepreneurial information search », Journal of Business Venturing, vol. 10, $\mathrm{n}^{\circ} 2$. 
COPIN, G. et S. MARION (1992), «Réinventer le business plan », Harvard Business Review, nº 65, été 1992, p. 96-102.

Dell'ANiello, P. (1987), Un plan d'affaires gagnant, Montréal, Publifor.

FILION, L.-J. (1991), Vision et relations : Clefs du succès de l'entrepreneur, Montréal, Éditions de l'Entrepreneur.

GARTNER, W.B. (1989), «Who is an entrepreneur? is the wrong question », Entrepreneurship Theory and Practice, vol. 13, $\mathrm{n}^{\circ} 4$, p. 47-67.

GIORDANO, Y. (1994), Du changement organisationnel à l'action stratégique, thèse, Université de Nice, IAE.

Guth, W.D., A. KumaraswamY et M. MCERLEAN (1991), «Cognition, enactment and learning in the entrepreneurial process », Frontiers of Entrepreneurship Research, p. 242-253.

HILLS, G.E. (1985), « Market analysis in the business plan : venture capitalists' perceptions », Journal of Small Business Management, janvier, p. 38-46.

HERNANDEZ, E.M. (1995), «L'entrepreneuriat comme processus », Revue internationale $P M E$, vol. 8, $\mathrm{n}^{\circ} 1$.

Julien, P.-A., dir. (1994), Les PME : Bilan et perspectives, Paris, Economica.

Julien, P.-A. et M. MARCheSnAY (1996), L'entrepreneuriat, Paris, Economica, coll. «Gestion Poche ».

KOENIG, G. (1994), «L'apprentissage organisationnel : repérage des lieux », Revue Française de Gestion, $\mathrm{n}^{\circ}$ 97, janvier-février, p. 76-83.

KOTLER, P. (1986), « Megamarketing », Harvard Business Review, $\mathrm{n}^{\circ}$ 42, automne 1986, p. 18-27.

Kotler, P. et P.L. DuboIS (1994), Marketing Management, Paris, Publi Union.

LABARBerA, P.-A. et S.A. Rosenberg (1989), "Marketing research and small entrepreneurial enterprises », Proceedings of Research Symposium on the Marketing / Entrepreneurship Interface, Chicago, p. 233-245.

LE Moigne, J.L. (1990), Théorie du système général, Paris, Presses universitaires de France.

MacClaughlin, H. (1985), Building your Business Plan : A Step by Step Approach, New York, John Wiley and Sons.

MaCMillan, I. (1985), «Les défis du créateur d'entreprise », Harvard, L'Expansion, hiver.

MARCHESNAY, M. (1993), Management stratégique, Paris, Eyrolles.

MARTINET, A.CH. (1984), Management stratégique : Organisation et politique, Paris, Édiscience International.

MARTINET, A.CH. (1988), «Le discours sur la stratégie d'entreprise», Revue Française de Gestion, $\mathrm{n}^{\circ}$ 67, janvier-février, p. 49-60.

MARTINET, A.CH. (1993), «Stratégie et pensée complexe », Revue Française de Gestion, mars-mai, $\mathrm{n}^{\circ} 93$, p. 64-72.

MORIN, E. (1982), Science avec conscience, Paris, Seuil, coll. « Points». 
PAPIN, R. (1995), Stratégie pour la création d'entreprise, Paris, Dunod.

PerrouX, F. (1973), Pouvoir et économie, Paris, Bordas.

PORTER, M. (1982), Choix stratégiques et Concurrence, Paris, Economica.

PORTER, M. (1986), L'avantage concurrentiel, Paris, Interéditions.

PORTER, M. (1991), « Toward a dynamic theory of strategy », Strategic Management Journal, vol. 12, p. 95-117.

SCHWENK, C.R. (1988), « The cognitive perspective on strategic decision-making », Journal of Management Studies, 25, p. 41-55.

Watlzlawick, P. et Collectif (1988), L'invention de la réalité : Contribution au constructivisme, Paris, Seuil, 366 p.

WEICK, K.E. (1969), The Social Psychology of Organizing, réédité en 1979, Reading, MA, Addison Wesley.

WeICK, K.E. (1995), Sensemaking in Organizations, Sage Publications, 231 p.

WEICK, K. et M. BougON (1986), «Organizations as cognitive maps : charting ways to success and failure », dans Sims et Gioia (dir.), The Thinking Organization, San Francisco, Jossey-Bass, p. 102-135.

YIN, R.K. (1989), Case Study, Research : Design and Methods, Newbury Park, CA, Sage Publications. 